typeset using JPSJ.sty $<$ ver.0.8 $>$

\title{
Effects of Umklapp Scattering on Electronic States in One Dimension
}

\author{
Masakazu Murakami 6 and Hidetoshi Fukuyama \\ Department of Physics, Tokyo University, Tokyo 113
}

(Received March 28, 1997)

\begin{abstract}
The effects of Umklapp scattering on electronic states are studied in one spatial dimension at absolute zero. The model is basically the Hubbard model, where parameters characterizing the normal $(U)$ and Umklapp $(V)$ scattering are treated independently. The density of states is calculated in the t-matrix approximation by taking only the forward and Umklapp scattering into account. It is found that the Umklapp scattering causes the global splitting of the density of states. In the presence of sufficiently strong Umklapp scattering, a pole in the t-matrix appears in the upper half plane, signalling an instability towards the ' $G / 2-$ pairing' ordered state ( $G$ is the reciprocal lattice vector), whose consequences are studied in the mean field approximation. It turns out that this ordered state coexists with spin-density-wave state and also brings about Cooper-pairs. A phase diagram is determined in the plane of $V$ and electron filling $n$.
\end{abstract}

KEYWORDS: modified t-matrix approximation, Umklapp scattering, density of states, mean field approximation, $G / 2-$ pairing, $G / 2-\mathrm{SDW}$.

\section{$\S 1 . \quad$ Introduction}

The problems of the metal-insulator transition due to the short-range Coulomb repulsion in the lattice system (known as the Mott transition 1 ) ) have attracted much interest. The Mott transition is often examined based on the Hubbard mode(2) with the hopping integral $t$ and the on-site Coulomb repulsion $U$. At least qualitatively, this phenomenon can be understood: If $U / t$ is large, electrons are kept apart each other. Especially for a half-filled band (one electron per one lattice site), they get completely localized because it requires large energy comparable with $U$ for them to move to another lattice site. As a result, the charge excitation spectrum has a gap known as the Mott-Hubbard gap and the system becomes an insulator. On the other hand if $U / t$ is small, it is energetically favorable for electrons to spread into the energy band and the system behaves as a metal. Hence it is understood that both the Coulomb repulsion and the existence of discrete lattice

\footnotetext{
* e-mail address : murakami@watson.phys.s.u-tokyo.ac.jp
} 
are the keys to the Mott transition. It was Hubbard who first succeeded in describing the qualitative feature of such behaviors 2 . By using the Hubbard model, he showed that the Mott-Hubbard gap begins to appear as $U / t$ gets larger in the half-filled case.

If we consider the on-site Coulomb interaction $U$ term in the wave vector representation, we have not only normal processes (which conserve the total momentum of two scattering electrons) but also Umklapp processes. Since the latter processes originate from the periodicity of the lattice and stand for the large-momentum-transfer processes, they play an important role in the Mott transition.

In the space dimension $d=1$, the exact solutions of the Hubbard model have been obtained by using Bethe Ansatz method . It was proved that the charge excitation spectrum has a gap and the system becomes an insulator for a half-filled band for any strength of Coulomb interaction. In this method, however, it is not easy to obtain the physically clear-cut understanding of the origin of the Mott-Hubbard gap. On the other hand, it was shown by the bosonization scheme 1 ) that the Umklapp scattering produces this gap in a half-filled band5.6. Moreover, characteristic features of the charge excitations in one-dimensional doped Mott insulators, especially the crossover between the metallic Drude type of excitation and the Mott-Hubbard gap, have been clarified (f) by mapping the model first to the g-ology model, , 国, then to the phase Hamiltonian 9 ) and finally to the massive Thirring model. It has also been disclosed that the Mott-Hubbard gap and other associated anomalies (the divergence of the compressibility near half filling, etc.) originate from Umklapp processes and how the system begins to show anomalies as the system approaches half filling $6,0,10,11$.

The emergence of the Mott insulator has also been clarified in the case of $d=\infty$ and it was also shown that the density of states has a gap together with the existence of quasi-particle peak before the onset of Mott transition 12 ). In $d=\infty$, however, we cannot distinguish Umklapp processes from normal ones because of the irrelevance of the momentum conservation.

From this point of view, it will be important to clarify the roles of the Umklapp scattering especially near half filling. In this paper, we examine the effects of Umklapp processes in detail at absolute zero for the simplest $d=1$ case, bearing the application to the $2 \mathrm{D}$ case in mind.

In $\S 2$, we introduce the model Hamiltonian. In $\S 3$, we study the density of states based on the perturbation theory with a special emphasis on both the forward and Umklapp scattering in the t-matrix approximation. Since we found that the t-matrix has a pole in the upper half plane if the Umklapp term becomes dominant, we study the nature of this instability in the mean field approximation in $\S 4$. Another type of instability associated with Umklapp processes is studied here. $\S 5$ is devoted to conclusion and discussion.

In the present paper, we take unit of $\hbar=k_{B}=1$. 


\section{§2. Model Hamiltonian}

We start with the one-dimensional Hubbard model with nearest-neighbor hopping:

$$
H=-t \sum_{<i j>\sigma}\left\{c_{i \sigma}^{\dagger} c_{j \sigma}+(\text { h.c. })\right\}+U \sum_{i} n_{i \uparrow} n_{i \downarrow}-\mu \sum_{i} c_{i \sigma}^{\dagger} c_{i \sigma},
$$

where $t(>0)$ is the transfer integral, $c_{i \sigma}\left(c_{i \sigma}^{\dagger}\right)$ is the annihilation (creation) operator for the electron on the i-th site with spin $\sigma, U(>0)$ is the Coulomb repulsion and $\mu$ is the chemical potential.

We can classify normal and Umklapp processes by the Fourier transformation,

$$
\begin{gathered}
H=H_{0}+H^{i n t}, \\
H_{0}=\sum_{-G / 2<k<G / 2} \sum_{\sigma} \xi_{k} c_{k \sigma}^{\dagger} c_{k \sigma}, \\
\xi_{k}=\epsilon_{k}-\mu, \\
\epsilon_{k}=-2 t \cos k, \\
H^{i n t}=H_{N}^{i n t}+H_{U}^{i n t},
\end{gathered}
$$

where $N, R_{i}, G$ and $q$ (or $q-G$ ) are the number of lattice sites, the lattice site vector, the reciprocal lattice vector equal to $2 \pi$ and the total momentum of two electrons, respectively. Here the lattice constant is taken as unity. Each wave vector $k$ of the electron lies in the first Brillouin zone, $-G / 2<k<G / 2$. The cosine band is shown in Fig. 1 . The bandwidth $W$ is equal to $4 t$. $H_{N}^{i n t}$ stands for normal processes which conserve the total momentum $q$ of two electrons, and $H_{U}^{\text {int }}$ stands for Umklapp processes in which it differs by $G$. Since we are mainly interested in Umklapp processes, we set the magnitude of interaction in $H_{U}^{i n t}$ as an arbitrary parameter $V(>0)$. In this way, we examine the effects of normal and Umklapp scattering separately. 


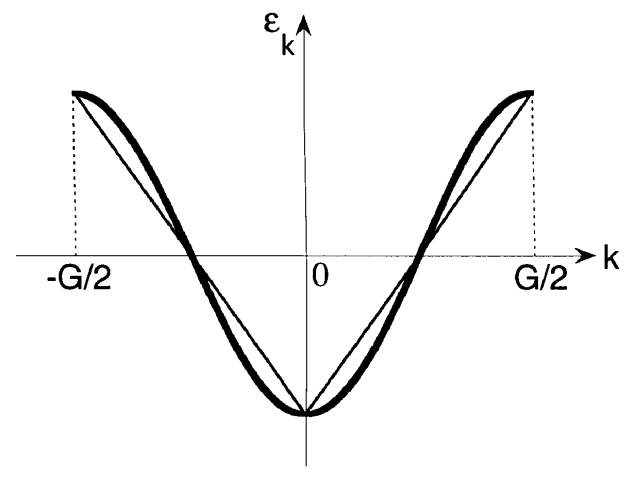

Fig. 1. Dispersion relation. Full and thin line show the cosine band and the linearized one, respectively.

\section{$\S 3 . \quad$ The Density of States in the Modified T-matrix Approximation}

In this section, we study the effects of the Umklapp scattering on the density of states based on the perturbation theory, at absolute zero $T=0$. For simplicity, we approximate the cosine dispersion with the liniarized one:

$$
\begin{aligned}
\epsilon_{k} & =v(|k|-G / 4), \\
\xi_{k} & =v\left(|k|-k_{F}\right),
\end{aligned}
$$

where $k_{F}$ is the Fermi wave vector and we put $v=8 t / G$ so that the bandwidth $W$ is left unchanged. In the half-filled case, $k_{F}$ is equal to $G / 4$. This dispersion is shown in Fig. 1.

We separate the dispersion into the branch with $k>0$ (right-moving) and with $k<0$ (leftmoving) and treat only the scattering between two electrons on the same branch - the forward and Umklapp scattering, as shown in Fig. 2. However, we neglect backward scattering processes in this paper, where the total momentum of two incident electrons is small.

(a)

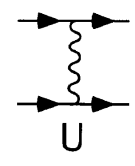

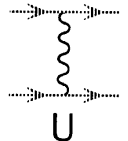

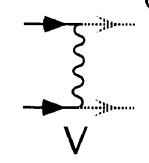

(b)

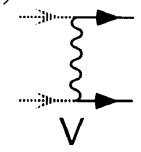

Fig. 2. (a) Forward and (b) Umklapp scattering processes. Full and dotted arrow lines stand for right-moving electron and left-moving electron, respectively. 


\subsection{Particle-Particle Correlation Function and the T-Matrix}

First of all we define the particle-particle correlation function $K\left(q, i \omega_{l}\right)$ with the total momentum q and energy $i \omega_{l}$ shown in Fig. 3,

$$
\begin{aligned}
K\left(q, i \omega_{l}\right) & =T \sum_{i \epsilon_{n}} \sum_{k} G_{0}\left(k, i \epsilon_{n}\right) G_{0}\left(-k+q,-i \epsilon_{n}+i \omega_{l}\right), \\
& =\sum_{k} \frac{f\left(\xi_{k}\right)-f\left(-\xi_{-k+q}\right)}{i \omega_{l}-\xi_{k}-\xi_{-k+q}}, \\
G_{0}\left(k, i \epsilon_{n}\right) & =\frac{1}{i \epsilon_{n}-\xi_{k}},
\end{aligned}
$$

where $G_{0}$ is the thermal Green function of $H_{0}, \omega_{l}=2 l \pi T$ and $\epsilon_{n}=(2 n+1) \pi T$ are the Matsubara frequencies and $f(\xi)$ is the Fermi distribution function. Since we consider only the interaction

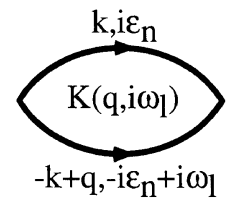

Fig. 3. Particle-particle correlation function, $K\left(q, i \omega_{l}\right)$.

between two electrons on the same branch, $\xi_{k}+\xi_{-k+q}$ is independent of $\mathrm{k}$ and we can calculate $K\left(q, i \omega_{l}\right)$ analytically. Especially, for $|q| \sim 2 k_{F}$ at absolute zero $T=0$,

$$
K(q, z)=-\frac{1}{2 \pi v} \frac{x_{q}}{z-x_{q}}
$$

where

$$
x_{q} \equiv v\left(|q|-2 k_{F}\right) .
$$

We note that $K(q, z)$ has one pole on the real axis $z=x_{q}$ and for $|q| \sim 2 k_{F}$ the residue is small.

Next we define the t-matrix $T\left(q, i \omega_{l}\right)$ without Umklapp processes (i.e., $\left.V=0\right)$,

$$
T\left(q, i \omega_{l}\right)=-\frac{U}{1+U K\left(q, i \omega_{l}\right)} .
$$

This is the particle-particle ladder diagrams shown in Fig. 4. By use of $K(q, z)$, eq. (3.3), we can calculate the t-matrix analytically. Especially for $|q| \sim 2 k_{F}$,

$$
T(q, z)=-U-U a \frac{x_{q}}{z-(1+a) x_{q}},
$$

where

$$
a \equiv \frac{U}{2 \pi v} \equiv \frac{U}{2 W}
$$

We note that $T(q, z)$ also has one pole on the real axis and for $|q| \sim 2 k_{F}$ the residue is small. 


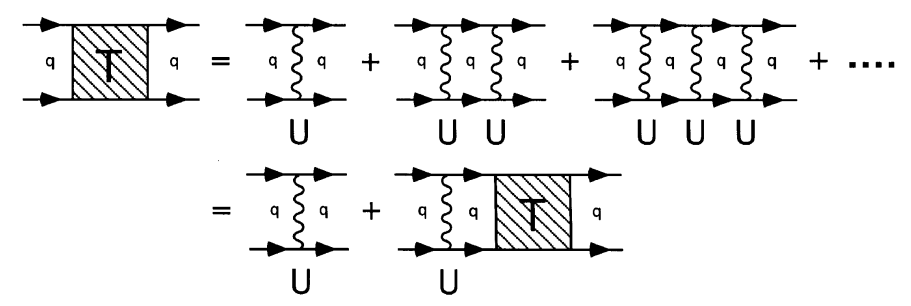

Fig. 4. The t-matrix, $T\left(q, i \omega_{l}\right)$.

\subsection{The Modified T-matrix}

We consider the particle-particle ladder diagram including Umklapp processes (i.e., $V \neq 0$ ). In this case the total momentum q of incident two electrons can be changed into $q-G(q>0)$ after the Umklapp scattering, i.e., the Umklapp interaction introduces the Bragg reflection of two incident electrons as a whole. In this case, the t-matrix is given as follows:

$$
\begin{gathered}
\hat{T} \equiv-\hat{U}-\hat{U} \hat{K} \hat{T} \\
\hat{T}=\left(\begin{array}{cc}
T_{11}(q, z) & T_{12}(q, z) \\
T_{21}(q, z) & T_{22}(q, z)
\end{array}\right), \\
\hat{U}=\left(\begin{array}{cc}
U & V \\
V & U
\end{array}\right), \\
\hat{K}=\left(\begin{array}{cc}
K(q, z) & 0 \\
0 & K(q-G, z)
\end{array}\right),
\end{gathered}
$$

where the index 1 stands for the total momentum $q>0$ and 2 for $q-G<0$. We call this matrix as a modified t-matrix. This is the particle-particle ladder diagram shown in Fig. 5. Especially for $q \sim 2 k_{F}, T_{11}(q, z)$ is given by

$$
\begin{gathered}
T_{11}(q, z)=-U-\frac{U}{x_{+}(q)-x_{-}(q)} \sum_{\gamma= \pm} \gamma \frac{\left(x_{\gamma}(q)-x_{q}\right)\left(x_{\gamma}(q)-c x_{q-G}\right)}{z-x_{\gamma}(q)}, \\
x_{ \pm}(q) \equiv \frac{1}{2}\left\{(1+a)\left(x_{q}+x_{q-G}\right) \pm \sqrt{(1+a)^{2}\left(x_{q}-x_{q-G}\right)^{2}+4 b^{2} x_{q} x_{q-G}}\right\} \\
c \equiv(1+a)-\frac{b^{2}}{a}, \quad b \equiv \frac{V}{2 \pi v} \equiv \frac{V}{2 W},
\end{gathered}
$$

and has two poles $z=x_{ \pm}(q)$. In the limit $V \rightarrow 0, T_{11}(q, z)$ reduces to eq. (3.6).

In the half-filled case $\left(G=4 k_{F}\right), x_{q-G}=-x_{q}$ and then

$$
x_{ \pm}(q)= \pm \alpha x_{q}, \quad \alpha \equiv \sqrt{(1+a)^{2}-b^{2}}
$$




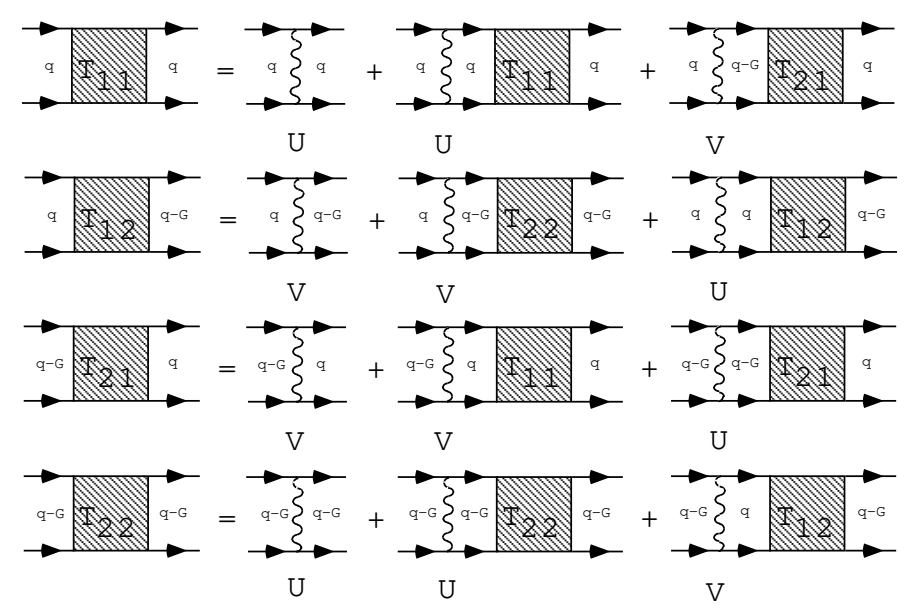

Fig. 5. The modified t-matrix, $\hat{T}\left(q, i \omega_{l}\right)$.

and therefore $T_{11}(q, z)$ for $q \sim 2 k_{F}$ is written as

$$
T_{11}(q, z)=-U-U\left\{\frac{(\alpha-1)(\alpha+c)}{2 \alpha} \frac{x_{q}}{z-\alpha x_{q}}-\frac{(\alpha+1)(\alpha-c)}{2 \alpha} \frac{x_{q}}{z+\alpha x_{q}}\right\} .
$$

The renormalization factor $\alpha$ of the velocity $v$ becomes pure imaginary if $b>1+a$, i.e., $V>U+2 W$. Therefore a pole appears in the upper half plane in this case. This fact implies that if the Umklapp interaction is sufficiently strong, the system becomes unstable. We will study this instability in the next section. In this section, $\alpha$ is assumed to be real.

\subsection{The Density of States}

In this section, we calculate the density of states. First of all, the single-particle spectral weight, $\rho(k, \epsilon)$, is given by

$$
\begin{aligned}
\rho(k, \epsilon) & =-\frac{1}{\pi} \operatorname{Im} G^{R}(k, \epsilon), \\
& =\rho_{c}(k, \epsilon)+\rho_{p}(k, \epsilon),
\end{aligned}
$$

where $G$ is the full Green function with the proper self energy $\Sigma, G^{R}(k, \epsilon)=\left[\epsilon-\xi_{k}-\Sigma^{R}(k, \epsilon)\right]^{-1}$. The superscript $\mathrm{R}$ stands for the retarded function. In eq. (3.12), $\rho_{c}(k, \epsilon)$ stands for the contribution from continuum part with $\operatorname{Im} \Sigma^{R}(k, \epsilon) \neq 0$,

$$
\rho_{c}(k, \epsilon)=-\frac{1}{\pi} \frac{\operatorname{Im} \Sigma^{R}(k, \epsilon)}{\left\{\epsilon-\xi_{k}-\operatorname{Re} \Sigma^{R}(k, \epsilon)\right\}^{2}+\left\{\operatorname{Im} \Sigma^{R}(k, \epsilon)\right\}^{2}},
$$

and $\rho_{p}(k, \epsilon)$ for the contribution from the poles with $\operatorname{Im} \Sigma^{R}(k, \epsilon)=0$,

$$
\rho_{p}(k, \epsilon)=\delta\left(\epsilon-\xi_{k}-\operatorname{Re} \Sigma^{R}(k, \epsilon)\right),
$$




$$
=\sum_{i} z_{i}(\epsilon) \delta\left(k-k_{i}(\epsilon)\right)
$$

where

$$
z_{i}(\epsilon)=\left|\frac{\partial}{\partial k}\left[\xi_{k}+\operatorname{Re} \Sigma^{R}(k, \epsilon)\right]\right|_{k=k_{i}(\epsilon)}^{-1},
$$

and $k_{i}(\epsilon)(\mathrm{i}=0,1,2, \cdots)$ is the solution of $\epsilon-\xi_{k}-\operatorname{Re} \Sigma^{R}(k, \epsilon)=0$ satisfying $\operatorname{Im} \Sigma^{R}(k, \epsilon)=0$ for given $\epsilon$. The summation is taken over all $k_{i}(\epsilon)$.

By use of $\rho(k, \epsilon)$, we obtain the density of states $\rho(\epsilon)$ per lattice site and spin,

$$
\rho(\epsilon)=\rho_{c}(\epsilon)+\rho_{p}(\epsilon)
$$

where

$$
\rho_{\nu}(\epsilon)=\frac{1}{N} \sum_{k} \rho_{\nu}(k, \epsilon)
$$

and $\nu=c, p$. In our approximation where the scattering processes between two electrons on the same branch are taken into account with the linear dispersion eq. (3.1), we can calculate $\Sigma^{R}(k, \epsilon)$ analytically by use of the t-matrix in both cases of $V=0$ and $V \neq 0$.

\subsubsection{The 2nd Order Perturbation in $U$}

First of all, we analyze the results in the 2 nd order perturbation in $U$ but $V=0$. In this case, the self energy can be written with $K(q, z)$ as follows:

$$
\Sigma\left(k, i \epsilon_{n}\right)=-T U^{2} \sum_{i \omega_{l}} \sum_{q} G_{0}\left(-k+q,-i \epsilon_{n}+i \omega_{l}\right) K\left(q, i \omega_{l}\right)
$$

After straightforward calculations, we obtain for $k>0$,

$$
\Sigma^{R}(k, \epsilon)=\left\{\begin{array}{cc}
\frac{1}{2} a^{2} \frac{\xi_{k}^{2}}{\epsilon-\xi_{k}+i \delta} & 0<k<2 k_{F}, \\
\frac{1}{2} a^{2} \frac{\xi_{k}^{2}-\xi_{k-k_{F}}^{2}}{\epsilon-\xi_{k}+i \delta} & 2 k_{F}<k<G / 2 .
\end{array}\right.
$$

Therefore it is seen that the Green function has two poles at $\epsilon=E_{ \pm}(k)$,

$$
E_{ \pm}(k)=\left\{\begin{array}{cl}
\left(1 \mp \frac{a}{\sqrt{2}}\right) \xi_{k} & \text { for } 0<k<k_{F} \\
\left(1 \pm \frac{a}{\sqrt{2}}\right) \xi_{k} & \text { for } k_{F}<k<2 k_{F} \\
\xi_{k} \pm a \sqrt{k_{F}\left(k-3 k_{F} / 2\right)} & \text { for } 2 k_{F}<k<G / 2 .
\end{array}\right.
$$

These energy bands for $k>0$ are shown in Fig. 6 . In the half-filled case, $2 k_{F}=G / 2$ and it is seen from eq. (3.20) that these dispersions are linear in $k$ over the first Brillouin zone.

The density of states consists only of the contribution from the poles, $\rho_{p}(k, \epsilon)$, which is shown in Fig. 7 for the half-filled case with $U / W=2.0$. 


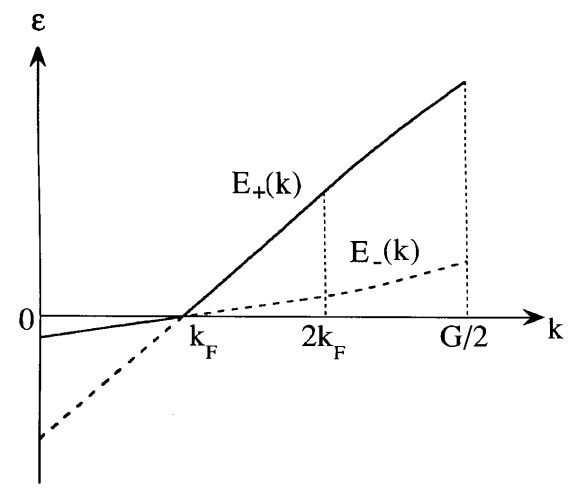

Fig. 6. The quasiparticle energy bands relative to the renormalized chemical potential in the 2nd order perturbation in $U$ for $k>0$ and less-than-half-filled case. The states with $\epsilon<0$ are occupied.

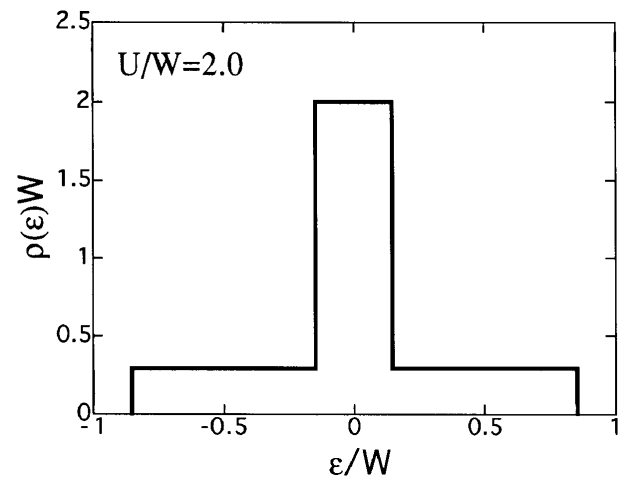

Fig. 7. The density of states in the second order perturbation in $U$ for the case of half filling and $U / W=2.0$.

\subsubsection{The T-matrix Approximation for $U$}

Next we study the density of states in the t-matrix approximation for $U$ in the absence of $V$. In this case, the self energy can be written with $T(q, z)$ as follows:

$$
\Sigma\left(k, i \epsilon_{n}\right)=-T \sum_{i \omega_{l}} \sum_{q} G_{0}\left(-k+q,-i \epsilon_{n}+i \omega_{l}\right) T\left(q, i \omega_{l}\right)
$$

Especially for $0<k<2 k_{F}, \Sigma^{R}(k, \epsilon)$ is given by

$$
\begin{gathered}
\operatorname{Re} \Sigma^{R}(k, \epsilon)=-a \xi_{k}-\left(\epsilon-\xi_{k}\right) \ln \left|\frac{\epsilon-(1+a) \xi_{k}}{\epsilon-\xi_{k}}\right|, \\
\operatorname{Im} \Sigma^{R}(k, \epsilon)=-\pi\left(\epsilon-\xi_{k}\right) \operatorname{sgn}\left(\xi_{k}\right) \text { for } 1<\epsilon / \xi_{k}<1+a .
\end{gathered}
$$


Here the contribution from the first term of the r.h.s. of (3.6) is neglected, because this can be included in the chemical potential shift. It is seen that the Green function $G^{R}(k, \epsilon)$ has two poles at $\epsilon=E_{ \pm}(k)$,

$$
E_{ \pm}(k)= \begin{cases}c_{\mp} \xi_{k} & \text { for } 0<k<k_{F} \\ c_{ \pm} \xi_{k} & \text { for } k_{F}<k<2 k_{F}\end{cases}
$$

where $c_{ \pm}$

$$
\begin{gathered}
c_{+}=1+1.19 a, \\
c_{-}=1-0.466 a .
\end{gathered}
$$

are determined numerically. There are two quasiparticle energy bands corresponding to these poles, which are shown for $0<k<2 k_{F}$ in Fig. 8 .

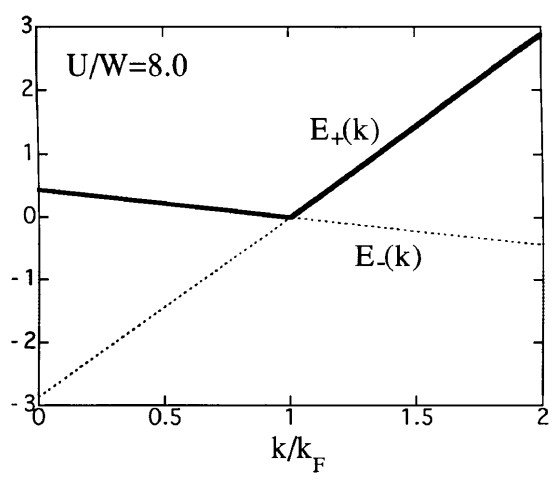

Fig. 8. The quasiparticle energy bands (scaled by $W$ ) relative to the renormalized chemical potential in the t-matrix approximation for $0<k<2 k_{F}$ and $U / W=8.0$. The states with $\epsilon<0$ are occupied.

The resultant density of states, $\rho_{c}(\epsilon)$ and $\rho_{p}(\epsilon)$ in the half-filled case is shown in Fig. 9 for $U / W=8.0$.

In the t-matrix approximation, there exists the contribution to the spectral weight also from the continuum part, i.e., $\rho_{c}(k, \epsilon) \neq 0$, although its contribution to the density of states, $\rho_{c}(\epsilon)$, is small. It is seen that $\rho_{p}(\epsilon)$ has appreciable spectral weight near the Fermi level $\epsilon \sim 0$. We note that for $U / W>>1, \rho(\epsilon) \neq 0$ over the range of $|\epsilon| \lesssim U / 4$.

\subsubsection{The Modified T-matrix Approximation}

Finally, we include Umklapp processes by use of $T_{11}(q, z)$. The modified t-matrix approximation leads to $\Sigma\left(k, i \epsilon_{n}\right)$ for $k>0$ as given by

$$
\Sigma\left(k, i \epsilon_{n}\right)=-T \sum_{i \omega_{l}} \sum_{q} G_{0}\left(-k+q,-i \epsilon_{n}+i \omega_{l}\right) T_{11}\left(q, i \omega_{l}\right)
$$




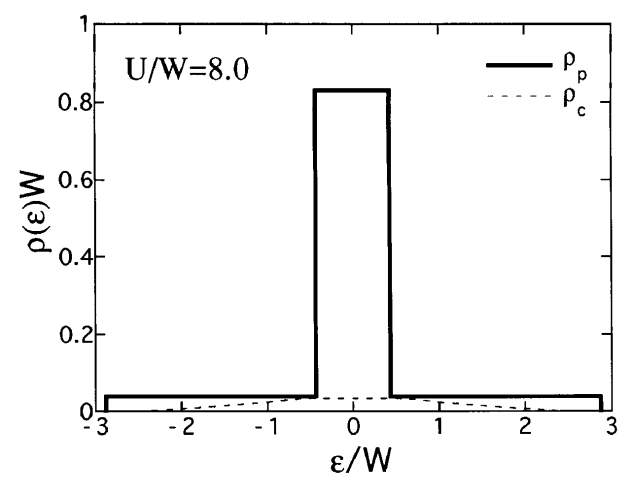

Fig. 9. The density of states in the t-matrix approximation for the case of half filling and $U / W=8.0$.

In this section, we will consider only the case of half filling for simplicity. When we calculate $\Sigma\left(k, i \epsilon_{n}\right)$ for $k>0$, we take only the account of contributions of the $T_{11}(q, i \omega l)$ with $q \sim 2 k_{F}$. After straightforward calculations, we can evaluate $\Sigma^{R}(k, \epsilon)$ and then $\rho(k, \epsilon)$. (The first term of the r.h.s. of (3.11) is included in the chemical potential shift.) There are three quasiparticle energy bands corresponding to the poles of $G^{R}(k, \epsilon)$. These are shown in Fig. 10 for $k>0$ and $U / W=V / W=8.0$. The Umklapp scattering produces the splitting between the upper and lower band, between which there exists another energy band.

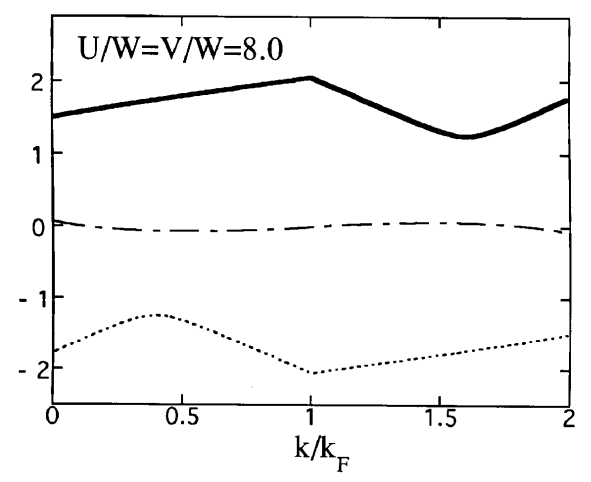

Fig. 10. The quasiparticle energy bands (scaled by $W$ ) relative to the renormalized chemical potential in the modified t-matrix approximation for the case of half filling, $k>0$ and $U / W=V / W=8.0$. The states with $\epsilon<0$ are occupied.

The density of states is shown in Fig. 11 for $U / W=8.0$ and a few values of $V / W$. It is seen that 
for $V \neq 0, \rho(\epsilon)$ has three-peak-structure. As the Umklapp scattering becomes strong, the upper and lower bands approach the Fermi level and the weight in these bands increases, while the weight in the middle peak near the Fermi level decreases.

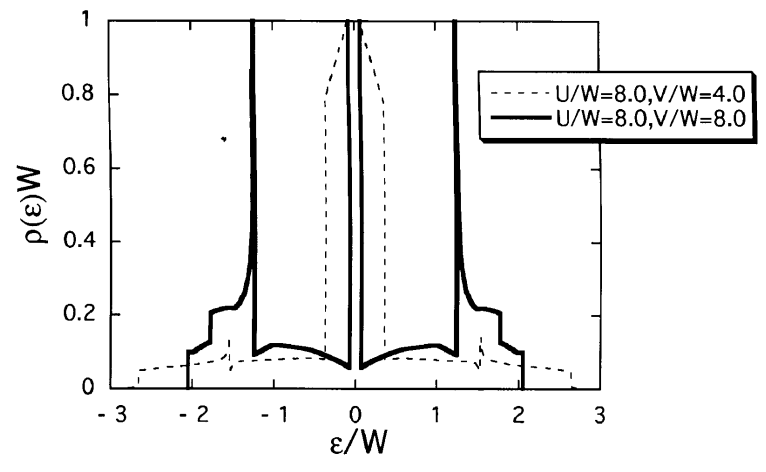

Fig. 11. The density of states in the half-filled case in the modified t-matrix approximation for $U / W=8.0$ and $V / W=4.0,8.0$.

The density of states in the case of $U=V$ is shown in Fig. 12. As $U$ or $V$ grows up in this case, the upper and lower band move away from the Fermi level and three-peak-structure becomes prominent. In this case, for $U / W>>1, \rho(\epsilon) \neq 0$ over the range of $|\epsilon| \lesssim \sqrt{U} / 2$.

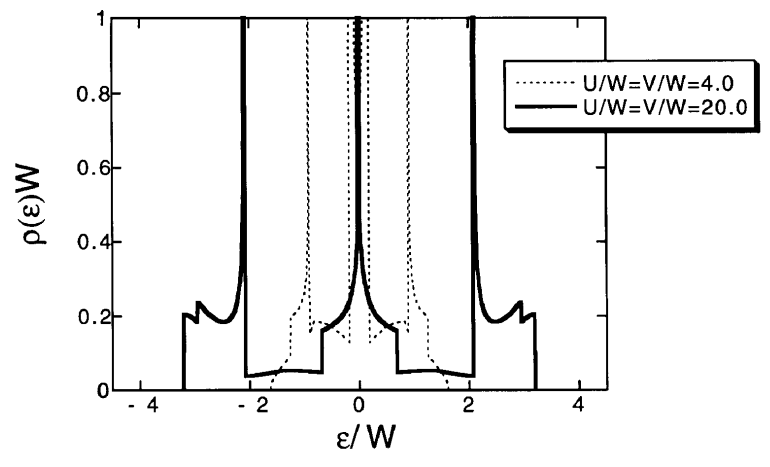

Fig. 12. The density of states in the modified t-matrix approximation for the case of half filling and $U / W=V / W=$ $4.0,20.0$.

As seen, Umklapp processes introduce the global splitting of the density of states, although there remains the appriciable spectral weight in the middle of split peaks and we cannot describe the 
Mott insulator.

We note that we calculated the self energy by the perturbation theory with the bare Green function $G_{0}$. In this calculation, the system behaves like the Fermi liquid in that the renormalization factor $Z \neq 0$. We consider that this is due to the lack of the self-consistency.

But we emphasize that we are interested in the global feature of the density of states, $\rho(\epsilon)$, as a function of $\epsilon$ in the half-filled case in the presence of the forward and Umklapp scattering.

\section{§4. Mean Field Approximation}

In the last section, we found that if the Umklapp scattering becomes dominant, there appears a pole in the upper half plane in the modified t-matrix. In this section, we examine this instability in the mean field approximation.

We use the cosine band eq. (2.2d $)$ as the dispersion. Hereafter, for simplicity, we set $U=0$ and consider $V$ only, and examine the ground state properties at absolute zero, $T=0$.

\subsection{The $G / 2-$ pairing}

The fact that there exists an instability in the particle-particle channel with the total momentum $|q| \sim 2 k_{F}$ naturally introduces the following order parameters,

$$
\begin{aligned}
\Delta_{R} & \equiv \frac{V}{N} \sum_{k}^{\prime}<c_{-k+G / 2 \downarrow} c_{k \uparrow}>, \\
\Delta_{L} & \equiv \frac{V}{N} \sum_{k}^{\prime}<c_{-k \downarrow} c_{k-G / 2 \uparrow}>,
\end{aligned}
$$

where

$$
\sum_{k}^{\prime} \equiv \sum_{0<k<G / 2}
$$

$\Delta_{R}\left(\Delta_{L}\right)$ is the particle-particle pairing with the total momentum $G / 2(-G / 2)$. In the half-filled case, $G / 2$ is equal to $2 k_{F}$.

We extract $q=G / 2$ term from $H_{U}^{\text {int }}$, which is decoupled to obtain the mean field Hamiltonian,

$$
\begin{gathered}
H^{M F}=\sum_{\alpha=R, L} H_{\alpha}+H_{c} \\
H_{\alpha}=\sum_{k}^{\prime} \psi_{k \alpha}^{\dagger} M_{k \alpha} \psi_{k \alpha}, \\
H_{c}=-\frac{2}{V} \operatorname{Re}\left(\Delta_{R} \Delta_{L}^{*}\right)+\sum_{k}^{\prime}\left(\xi_{-k+G / 2}+\xi_{-k}\right), \\
\psi_{k R}=\left(\begin{array}{c}
c_{k \uparrow} \\
c_{-k+G / 2 \downarrow}^{\dagger}
\end{array}\right), \psi_{k L}=\left(\begin{array}{c}
c_{k-G / 2 \uparrow} \\
c_{-k \downarrow}^{\dagger}
\end{array}\right), \\
M_{k R}=\left(\begin{array}{cc}
\xi_{k} & \Delta_{L} \\
\Delta_{L}^{*} & -\xi_{-k+G / 2}
\end{array}\right), M_{k L}=\left(\begin{array}{cc}
\xi_{k-G / 2} & \Delta_{R} \\
\Delta_{R}^{*} & -\xi_{-k}
\end{array}\right) .
\end{gathered}
$$


We note that there exists a self-consistent solution only for the case of $\Delta_{R}=-\Delta_{L} \equiv \Delta_{1}$.

The quasiparticle dispersion in this case is given by

$$
\begin{gathered}
E_{\alpha}^{ \pm}(k)=c_{\alpha} \epsilon_{k} \pm E_{g}, \\
E_{g} \equiv \sqrt{\mu^{2}+\left|\Delta_{1}\right|^{2}},
\end{gathered}
$$

where $c_{\alpha}=1(-1)$ for $\alpha=R(L)$. This is shown in Fig. 13(a).
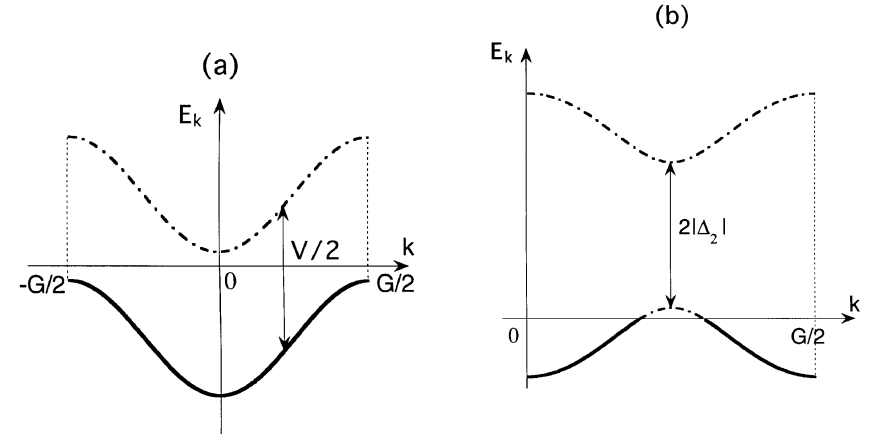

(c)

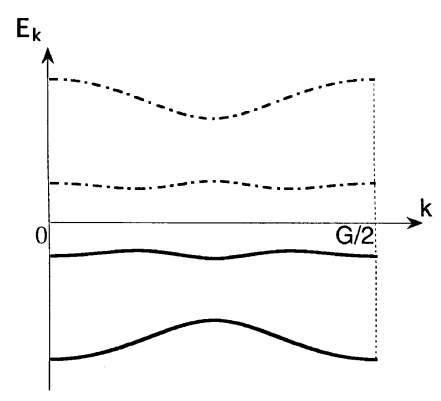

Fig. 13. The quasiparticle energy bands relative to the chemical potential for (a) $G / 2$-pairing state, (b) $G / 2-$ SDW state and (c) the coexisting state with both $G / 2-$ pairing and $G / 2-$ SDW. Full line shows the occupied state.

It is seen that $\Delta_{1} \neq 0$ only for $V>8 t$, in which case we have

$$
\begin{aligned}
\mu & =\frac{V}{4}(n-1), \\
\left|\Delta_{1}\right| & =\frac{V}{4} \sqrt{n(2-n)},
\end{aligned}
$$

where $n(0<n<2)$ is the filling (the expectation value of the number of electrons) per lattice site. (There is another solution for $8 t<V<12 \pi t$, although it corresponds to a local maximum of the energy.) It turns out from eq. (4.4) and (4.5) that $E_{g} \equiv V / 4$ for $V>8 t$ and the shape of the energy band does not change for arbitrary filling $n$ and the Fermi level lies always in the energy gap. Such a state is possible, because the weight of the lower band shifts into the upper band as $n$ decreases. If we use the linear dispersion, eq. (3.1), nonzero $\Delta_{1}$ exists for $V>2 \pi v=2 W$. Therefore it is seen that this corresponds to the condition that the modified t-matrix has a pole in the upper half plane. The essential condition for the stability of this ordered state is $\epsilon_{k}+\epsilon_{-k+G / 2}=0$.

The total energy per lattice site, $E_{1}$, for fixed $n$ is

$$
E_{1}=\frac{V}{8} n(n-2)
$$

We note that the existence of $\Delta_{1}$ does not lead to the $\eta$-pair condensation 13,14 , since

$$
\hat{O}_{\eta-\text { pair }} \equiv \frac{1}{N} \sum_{i}(-1)^{i} c_{i \uparrow} c_{i \downarrow}
$$




$$
\begin{aligned}
<\hat{O}_{\eta-\text { pair }}> & =-\frac{1}{V}\left(\Delta_{R}+\Delta_{L}\right) \\
& \equiv 0
\end{aligned}
$$

However the following type of coherence does exist,

$$
\begin{aligned}
\left\langle c_{j \uparrow} c_{j \downarrow}>=\right. & -i \frac{\sqrt{n(2-n)}}{\pi} \frac{(-1)^{j}}{j-j \prime} \\
& \text { for } j \prime=j \pm 1, j \pm 3, \cdots .
\end{aligned}
$$

The $n$-dependences of $\left|\Delta_{1}\right|, \mu$ and $E_{1}$ are shown for $V / t=12$ in Fig. 14.

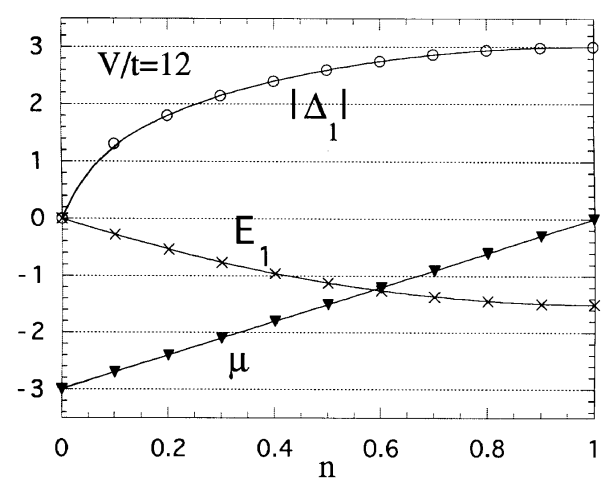

Fig. 14. The dependences of $\left|\Delta_{1}\right|$, chemical potential, $\mu$, and total energy, $E_{1}$, (scaled by $t$ ) on the electron density in $G / 2-$ pairing state for $V / t=12$.

\subsection{The $G / 2-S D W$}

Since two electrons with $k \sim k_{F}$ (or $\left.-k_{F}\right)$ are scattered into the final state with $k \sim-k_{F}\left(\right.$ or $\left.k_{F}\right)$, the following particle-hole pairing order parameters are expected to be stabilized:

$$
\Delta_{\sigma} \equiv \frac{V}{N} \sum_{k}^{\prime}<c_{k-G / 2 \sigma}^{\dagger} c_{k \sigma}>
$$

where $\sigma=\uparrow, \downarrow$ and $\sum_{k}^{\prime} \equiv \sum_{0<k<G / 2}$. The mean field Hamiltonian by using these order parameters is given as follows,

$$
\begin{gathered}
H^{M F}=\sum_{\sigma} H_{\sigma}+H_{c}, \\
H_{\sigma}=\sum_{k}^{\prime} \psi_{k \sigma}^{\dagger} M_{k \sigma} \psi_{k \sigma}, H_{c}=-\frac{2}{V} \operatorname{Re}\left(\Delta_{\uparrow} \Delta_{\downarrow}\right), \\
\psi_{k \sigma}=\left(\begin{array}{c}
c_{k \sigma} \\
c_{k-G / 2 \sigma}
\end{array}\right), M_{k \sigma}=\left(\begin{array}{cc}
\xi_{k} & \Delta_{\bar{\sigma}}^{*} \\
\Delta_{\bar{\sigma}} & \xi_{k-G / 2}
\end{array}\right),
\end{gathered}
$$


where $\bar{\sigma}$ stands for the spin opposite to $\sigma$.

Here we take $\Delta_{\uparrow}=-\Delta_{\downarrow}^{*} \equiv \Delta_{2}$ so that there exists a self-consistent solution. We write $\Delta_{2}=$ $\left|\Delta_{2}\right| e^{i \theta}$ where $0 \leq \theta \leq \pi / 2$. As we shall see later, this phase factor is associated with the amplitude of the spin-density-wave (SDW) order parameter.

The quasiparticle dispersion given by eq. (4.10) is as follows,

$$
\begin{aligned}
& E_{ \pm}(k)= \pm E(k)-\mu, \\
& E(k) \equiv \sqrt{\epsilon_{k}^{2}+\left|\Delta_{2}\right|^{2}} .
\end{aligned}
$$

These are shown in Fig. 13(b). In this case the energy gap is $2\left|\Delta_{2}\right|$.

We can determine $\Delta_{2}$ numerically from the self-consistent equation,

$$
1=\frac{V}{2 \pi} \int_{0}^{\frac{\pi}{2}(1-|1-n|)} \frac{d k}{E(k)},
$$

and the chemical potential $\mu$ is obtained by using this $\Delta_{2}$,

$$
\mu=\operatorname{sgn}(n-1) \sqrt{\left|\Delta_{2}\right|^{2}+4 t^{2} \cos ^{2}\left(\frac{\pi}{2} n\right)} .
$$

This solution exists for $n_{c 2}<n<2-n_{c 2}$, where

$$
n_{c 2}=\frac{2}{\pi} \sin ^{-1}\left[\tanh \left(\frac{4 \pi t}{V}\right)\right]
$$

We see from eq. (4.12) that in the half-filled case $(n=1)$ this solution exists once $V>0$. In the limit $(V / t=\infty)$, the energy gap is equal to $V / 2$. The nesting property $\epsilon_{k}+\epsilon_{k-G / 2}=0$ is again crucial for the presence of this order parameter.

The total energy per lattice site, $E_{2}$, for fixed $n$ is

$$
E_{2}=-\frac{2}{\pi} \int_{0}^{\frac{\pi}{2}(1-|1-n|)} E(k) d k+2 \frac{\left|\Delta_{2}\right|^{2}}{V} .
$$

In accordance with nonzero $\Delta_{2}$, the long range order of SDW is induced, but not that of CDW, because

$$
\begin{aligned}
\hat{O}_{C D W} & \equiv \frac{1}{N} \sum_{i \sigma}(-1)^{i} c_{i \sigma}^{\dagger} c_{i \sigma}, \\
\hat{O}_{S D W} & \equiv \frac{1}{2 N} \sum_{i \sigma}(-1)^{i} \sigma c_{i \sigma}^{\dagger} c_{i \sigma}, \\
<\hat{O}_{C D W}> & =\frac{2}{V} \operatorname{Re}\left(\Delta_{\uparrow}+\Delta_{\downarrow}\right), \\
& \equiv 0 \\
<\hat{O}_{S D W}> & =\frac{1}{V} \operatorname{Re}\left(\Delta_{\uparrow}-\Delta_{\downarrow}\right), \\
& \equiv 2 \frac{\left|\Delta_{2}\right|}{V} \cos \theta .
\end{aligned}
$$


We note that the system is degenerate with respect to $\theta$ and in the case $\theta=\pi / 2$ the SDW order vanishes.

Recently Wilczek et.al. have introduced the same order parameter 15 :

$$
<c_{k+G / 2 \sigma}^{\dagger} c_{k \sigma}>=\text { pure imaginary }
$$

which corresponds to $\theta=\pi / 2$ in the present notation, i.e., without SDW order. They showed that this order (which was named as 'd-Density order') exists in the 2-d extended Hubbard model with both on-site and nearest-neighbor Coulomb repulsion, and have discussed the possible relationship to the Mott insulators.

The $n$-dependences of $\left|\Delta_{2}\right|, \mu$ and $E_{2}$ are shown for $V / t=12$ in Fig. 15 . The important thing is

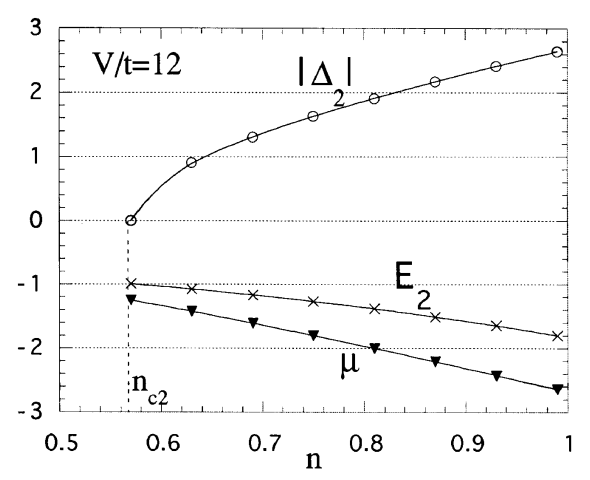

Fig. 15. The dependences of $\left|\Delta_{2}\right|$, chemical potential $\mu$ and total energy $E_{2}$ (scaled by $t$ ) on the electron density in $G / 2-$ SDW state for $V / t=12$. For $n>n_{c 2}\left|\Delta_{2}\right| \neq 0$. In the half-filled case, $\mu=0$.

that $E_{2}$ is decreasing function of $n$, i.e., the compressibility $\kappa$ is negative, $\kappa \equiv \partial n / \partial \mu<0$, except for the half-filled case $n=1$ at which $\mu$ changes discontinuously. Therefore there occurs phase separation between a electron-rich and a electron-free phase.

\subsection{The $G / 2$-pairing \& $G / 2-S D W$}

It is seen, in fact, that both $\Delta_{1}$ and $\Delta_{2}$ can coexist. To see this, we construct the mean-field Hamiltonian as follows:

$$
\begin{gathered}
H^{M F}=\sum_{k}^{\prime} \psi_{k}^{\dagger} M_{k} \psi_{k}+H_{c}, \\
\psi_{k}^{\dagger}=\left(c_{k \uparrow}^{\dagger} c_{-k+G / 2 \downarrow} c_{k-G / 2 \uparrow}^{\dagger} c_{-k \downarrow}\right),
\end{gathered}
$$




$$
\begin{gathered}
M_{k}=\left(\begin{array}{cccc}
\epsilon_{k}-\mu & -\Delta_{1} & -\Delta_{2} & 0 \\
-\Delta_{1}^{*} & \epsilon_{k}+\mu & 0 & -\Delta_{2} \\
-\Delta_{2}^{*} & 0 & -\epsilon_{k}-\mu & \Delta_{1} \\
0 & -\Delta_{2}^{*} & \Delta_{1}^{*} & -\epsilon_{k}+\mu
\end{array}\right), \\
H_{c}=\frac{2}{V}\left(\left|\Delta_{1}\right|^{2}+\left|\Delta_{2}\right|^{2}\right)-\mu .
\end{gathered}
$$

We note that, if both $\Delta_{1}$ and $\Delta_{2}$ are nonzero, the Cooper-pair also exists in general,

$$
\begin{aligned}
\hat{O}_{C} & \equiv \frac{1}{N} \sum_{i} c_{i \downarrow} c_{i \uparrow}, \\
<\hat{O}_{C}> & =\frac{1}{N} \sum_{-G / 2<k<G / 2}<c_{-k \downarrow} c_{k \uparrow}> \\
& \neq 0 .
\end{aligned}
$$

Hence it is necessary, in principle, to consider the Cooper-pairing term in the mean field Hamiltonian. In the present case of $U=0$, however, such a term is given by

$$
\begin{aligned}
H= & \frac{V}{N}\left\{c_{G / 2 \uparrow}^{\dagger} c_{G / 2 \downarrow}^{\dagger} \sum_{-G / 2<k<G / 2} c_{-k \downarrow} c_{k \uparrow}+(\text { h.c. })\right\}, \\
\rightarrow & <c_{G / 2 \uparrow}^{\dagger} c_{G / 2 \downarrow}^{\dagger}>\frac{V}{N} \sum_{-G / 2<k<G / 2} c_{k \downarrow} c_{-k \uparrow} \\
& +V<\hat{O}_{C}>c_{G / 2 \uparrow}^{\dagger} c_{G / 2 \downarrow}^{\dagger}+(\text { h.c. }),
\end{aligned}
$$

i.e., the term of the total momentum $q=0$ is associated only with that of $q=G$ in Umklapp processes. Since the term $q=G$ consists of only one state of two electrons, each of which has wave vector $G / 2$, this gives the correction of $O\left(\frac{1}{N}\right)$ to the total energy and we can neglect it.

In the presence of $\Delta_{1}$ and $\Delta_{2}$, the quasiparticle energies are given by $\pm E_{ \pm}(k)$,

$$
\begin{aligned}
& E_{ \pm}(k)=\sqrt{\epsilon_{k}^{2}+\mu^{2}+\left|\Delta_{1}\right|^{2}+\left|\Delta_{2}\right|^{2} \pm 2 A(k)} \\
& A(k) \equiv \sqrt{\epsilon_{k}^{2}\left|\Delta_{1}\right|^{2}+\mu^{2}\left(\epsilon_{k}^{2}+\left|\Delta_{2}\right|^{2}\right)}
\end{aligned}
$$

This is shown in Fig. 13(c).

The self-consistent equations in this case are

$$
\begin{aligned}
n & =1+\mu \int_{0}^{\pi / 2} \frac{d k}{\pi}\left\{\frac{\epsilon_{k}^{2}+\left|\Delta_{2}\right|^{2}}{A(k)}\left[\frac{1}{E_{+}(k)}-\frac{1}{E_{-}(k)}\right]+\left[\frac{1}{E_{+}(k)}+\frac{1}{E_{-}(k)}\right]\right\}, \\
\Delta_{1} & =V \Delta_{1} \int_{0}^{\pi / 2} \frac{d k}{4 \pi}\left\{\frac{\epsilon_{k}^{2}}{A(k)}\left[\frac{1}{E_{+}(k)}-\frac{1}{E_{-}(k)}\right]+\left[\frac{1}{E_{+}(k)}+\frac{1}{E_{-}(k)}\right]\right\}, \\
\Delta_{2} & =V \Delta_{2} \int_{0}^{\pi / 2} \frac{d k}{4 \pi}\left\{\frac{\mu^{2}}{A(k)}\left[\frac{1}{E_{+}(k)}-\frac{1}{E_{-}(k)}\right]+\left[\frac{1}{E_{+}(k)}+\frac{1}{E_{-}(k)}\right]\right\} .
\end{aligned}
$$


These are solved numerically. If there is a solution with $\Delta_{1}, \Delta_{2} \neq 0$, the correlation of the Cooper-pair, eq. (4.22), is obtained as follows:

$$
<\hat{O}_{C}>=-\frac{\mu}{V}\left|\Delta_{1}\right|\left|\Delta_{2}\right| \sin \theta \int_{0}^{\pi / 2} \frac{d k}{2 \pi} \frac{1}{A(k)}\left[\frac{1}{E_{+}(K)}-\frac{1}{E_{-}(k)}\right] .
$$

We note that the phase factor, $\theta$, associated with the amplitude of SDW order parameter, eq. (4.19), appears also in the correlation of the Cooper-pair.

The solution with $\Delta_{1}, \Delta_{2} \neq 0$ exists for $n_{c 1}<n<2-n_{c 1}$ if $V>8 t$, where

$$
n_{c 1} \equiv 1-\sqrt{1-\sqrt{1-\left(\frac{8 t}{V}\right)^{2}}} .
$$

In the limit of $V \rightarrow 8 t, n_{c 1} \rightarrow 0$ and $\Delta_{1} \rightarrow 0$. It is seen that for a half-filled band $n=1(\mu=0)$, this solution exists only in the limit $t=0$.

The total energy per lattice site, $E_{3}$, for fixed $n$ is

$$
E_{3}=-\int_{0}^{\pi / 2} \frac{d k}{\pi}\left[E_{+}(k)+E_{-}(k)\right]+\frac{2}{V}\left(\left|\Delta_{1}\right|^{2}+\left|\Delta_{2}\right|^{2}\right)+\mu(n-1) .
$$

The $n$-dependences of $\left|\Delta_{1}\right|,\left|\Delta_{2}\right|,\left|\Delta_{c}\right| \equiv V\left|<\hat{O}_{C}>\right|, \mu$ and $E_{3}$ are shown in Fig. 16 for $V / t=12$ and $\theta=\pi / 2$. In the case of $\Delta_{1}, \Delta_{2} \neq 0$, the compressibility $\kappa$ becomes positive in contrast to the case of $\Delta_{1}=0$ and $\Delta_{2} \neq 0$.

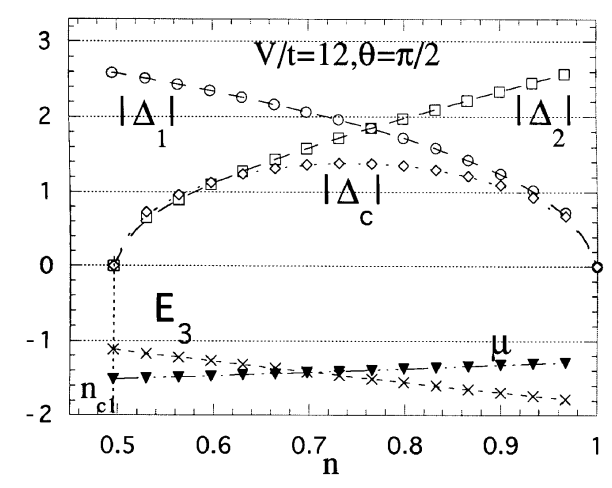

Fig. 16. The dependences of $\left|\Delta_{1}\right|,\left|\Delta_{2}\right|,\left|\Delta_{c}\right|$, chemical potential $\mu$ and total energy $E_{3}$ (scaled by $t$ ) on the electron density in the coexisting state with both $G / 2$-pairing and SDW for $V / t=12$ and $\theta=\pi / 2$. These two states coexist for $n_{c 1}<n<1$.

\subsection{Phase Diagram}

The state with the lowest energy has been determined in the $V / t-n$ plane for the region of $0 \leq n \leq 1$. The result is shown in Fig. 17 . 


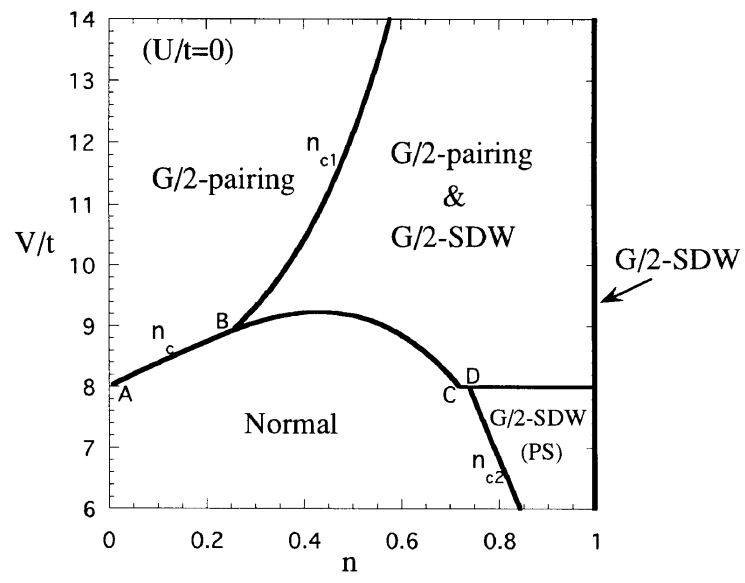

Fig. 17. Phase diagram in the plane of $V / t$ and $n$ for $U=0$. PS stands for phase separation. Line AB stands for $n_{c}$. Line BCD stands for the boundary between the normal state and the coexisting state with both $G / 2-$ pairing and $G / 2-\mathrm{SDW}$. For detailed explanations about $n_{c}, n_{c 1}$ and $n_{c 2}$, see the text.

To begin with, we consider the case $V / t>8$. We compare $G / 2$-pairing state with the normal state. By noting that the total energy of the normal state $E_{0}$ is equal to $-\frac{4 t}{\pi} \sin \left(\frac{\pi}{2} n\right)$ and comparing this with $E_{1}$, eq. (4.6), we see that the boundary between the normal state and the $G / 2$-pairing state is of the first order as $V$ is varied and is given as follows,

$$
\begin{array}{ll}
0<n<n_{c} & \text { for } 8 t<V<V_{c} \\
0<n \leq 1 & \text { for } V>V_{c}
\end{array}
$$

where $V_{c} / t$ is equal to $32 / \pi(\sim 10.2)$ and $n_{c}$ is determined as the solution of the following equation,

$$
\frac{V}{8} n_{c}\left(n_{c}-2\right)=-\frac{4 t}{\pi} \sin \left(\frac{\pi}{2} n_{c}\right) .
$$

Next we discuss $G / 2-\mathrm{SDW}$ state. The transition from the normal state to this state is of the second order as a function of $V$. As discussed in the last section, this state is unstable against the phase separation (except for the case of half filling). However, it is energetically favorable compared to the normal state, $E_{2}<E_{0}$ for $n_{c 2}<n \leq 1$, where $E_{2}$ and $n_{c 2}$ is given by eq. (4.15) and eq. (4.14), respectively. On the other hand, this state competes with $G / 2$-pairing state for $V>V_{c 1}$ and $n_{c 2}<n<n_{c 1}$, where $V_{c 1} / t \sim 13.0$ and $n_{c 1}$ is given by eq. (4.26). ( $V_{c 1}$ is determined as the solution of $n_{c 1}=n_{c 2}$.) It is seen that in this region $G / 2$-pairing state is stable, $E_{1}<E_{2}$.

In the coexisting state with both $G / 2$-pairing and $G / 2-\mathrm{SDW}$ for $V / t>8$ and $n_{c 1}<n<1$, the total energy, $E_{3}$, eq. (4.27), is always lower than that of the state with only one order parameter among them, i.e., $E_{3}<E_{1}, E_{2}$. On the other hand, for $V / t \sim 8$, the energy of this state is higher 
than that of the normal state, $E_{0}<E_{3}$, i.e., the transition from the normal state to this coexisting state is of the first order as a function of $V$. The boundary between the normal state and this coexisting state is determined numerically as the solution of $E_{0}=E_{3}$. It is seen that $E_{3}<E_{0}$, independent of $n$, for $V / t \gtrsim 9.23$. Especially in this region, the ground state is given as follows:

$$
\begin{array}{cc}
n=1 & G / 2-\mathrm{SDW}, \\
n_{c 1}<n<1 & G / 2-\text { pairing \& } G / 2-\mathrm{SDW}, \\
0<n<n_{c 1} & G / 2-\text { pairing, }
\end{array}
$$

On the other hand, for $0<V / t<8$, the ground state is given as follows:

$$
\begin{array}{cc}
n=1 & G / 2-\mathrm{SDW}, \\
0<n<n_{c 2} & \text { normal, }
\end{array}
$$

and for $n_{c 2}<n<1$ there occurs phase separation.

\section{$\S 5 . \quad$ Conclusion and Discussion}

We have studied the effects of the Umklapp scattering in the 1D Hubbard model by treating the strength of normal $(U)$ and Umklapp $(V)$ interaction as independent parameters, keeping the bandwidth $(W)$ finite.

We calculated the density of states by use of the perturbation theory with the linear dispersion with a special emphasis on both the forward and Umklapp scattering.

For $V=0$, we found that the t-matrix (the summation of the particle-particle ladder diagram with respect to $U$ ) has one pole on the real axis. The density of states, $\rho(\epsilon)$, in the t-matrix approximation, has appreciable spectral weight and no energy gap at the Fermi level in the halffilled case.

In the presence of $V$, we found that the modified t-matrix (including $V$ term) has two poles on the real axis for $0<V \leq U$. In the resultant $\rho(\epsilon)$ in the modified t-matrix approximation, the global splitting is introduced and three-peak-structure is observed in the half-filled case, although the appreciable spectral weight remains in the middle of split peaks near the Fermi level. As the Umklapp scattering becomes strong, this structure becomes prominent. However, we have not described the Mott insulator.

On the other hand, if $V>U+2 W$, a pole of the modified t-matrix appears in the upper half plane. Therefore in $\S 4$, first of all we studied this instability in the mean field approximation for $U=0$ and found that in the strong coupling regime, $G / 2$-pairing state becomes stable. Next we examined another instability associated with Umklapp processes and found that there appears G/2-SDW state. This state, however, is metastable due to the negative compressibility except for a half-filled band. Finally, we found that the above two order parameters can coexist.

We constructed a phase diagram in the full $V / t-n$ plane for these possible states. In the strong coupling regime $V / t \gtrsim 8$, the ground state is the $G / 2-$ SDW state at just half filling $n=1$, the 
coexisting state with both $G / 2$-pairing and $G / 2-$ SDW near half filling and the $G / 2$-pairing state at low filling. In this coexisting state near half filling, the correlation of the Cooper-pair appears and is connected with the SDW order parameter through one phase angle, $\theta$, i.e., $<\hat{O}_{C}>\propto \sin \theta$, eq. 4.25), and $<\hat{O}_{S D W}>\propto \cos \theta$, eq. (4.19), through the existence of the $G / 2-$ pairing. Such close relationship between the SDW and Cooper-pair has been indicated in the 2D $t-J$ model with the $\mathrm{SO}(5)$ symmetry 1 ), which unifies antiferromagnetism with d-wave superconductivity. The application of the present theory to such $2 \mathrm{D}$ case is the future problem.

\section{Acknowledgements}

M.M. would like to express his gratitude to Hiroshi Kohno for instructive discussions and suggestions. This work is financially supported by a Grant-in-Aid for Scientific Research on Priority Area "Anomalous Metallic State near the Mott Transition" (07237102) from the Ministry of Education, Science, Sports and Culture.

[1] N.F.Mott, Proc.Roy.Soc.Sci.A62(1949)416.

[2] J.Hubbard, Proc.Roy.Soc.(London)A 281(1964)401.

[3] E.H.Lieb and E.Y.Wu, Phys.Rev.Lett.20(1968)1445.

[4] For a review, V.J.Emery, in Highly Conducting One-Dimensional Solids, ed. by J.Devreese, R.Evrad and V.van Doren (Plenum, New York, 1979) P.24\%.

[5] V.J.Emery, A.Luther and I.Peschel, Phys.Rev.B13(1976) 1272

[6] T.Giamarchi, Phys.Rev.B44(1991)2905.

[7] M.Mori, H.Fukuyama and M.Imada, J.Phys.Soc.Jpn.63(1994)1639; M.Mori and H.Fukuyama, J.Phys.Soc.Jpn.65(1996)3604.

[8] For a review, J.Solyom, Adv.Phys.28(1979)201.

[9] For a review, H.Fukuyama and H.Takayama, Electronic Properties of Inorganic Quasi One-Dimensional Compounds Part 1 ed. by P.Monceau Reidel Pub.Comp,1985 P.41.

[10] V.J.Emery, Phys.Rev.Lett.65(1990)1076.

[11] H.J.Schulz, Phys.Rev.Lett.64(1990)2831.

[12] For a review, A.Georges, G.Kotliar, W.Krauth and M.J.Rozenberg, Rev.Mod.Phys.68(1996)13

[13] C.N.Yang, Phys.Rev.Lett.63(1989)2144.

[14] S.C.Zhang, Phys.Rev.Lett.65(1990)120.

[15] C.Nayak and F.Wilczek: cond-mat/9510132; F.Wilczek: cond-mat/9512156; C.Nayak and F.Wilczek: condmat/9602112.

[16] S.C.Zhang, Science: to be published. 